\title{
Circadian clocks and pregnancy
}

\author{
James Olcese* \\ Department of Biomedical Sciences, Florida State University College of Medicine, Tallahassee, FL, USA \\ ${ }^{*}$ Correspondence: james.olcese@med.fsu.edu
}

Edited and reviewed by:

Raul Miguel Luque, University of Cordoba, Spain

Keywords: circadian, circadian clocks, pregnancy, reproductive systems, cellular circadian clock

The recognition that 24 -h rhythmic processes ("circadian") underlie many endocrine functions has added a fascinating new temporal dimension to our appreciation of their complexity. Research from various laboratories has revealed circadian gene expression in multiple tissues in the reproductive system of non-pregnant and pregnant mammals. However, many questions regarding role of circadian clocks in the female reproductive system remain unresolved. This Research Topic on Circadian Clocks and Pregnancy brings together a broad and innovative group of researchers to discuss, evaluate, and summarize the key aspects of circadian function as it relates to all aspects of pregnancy from ovulation and implantation to fetal development and parturition.

In the initial review, Ditisheim et al. (1) address preeclampsia, a pregnancy-related disease that involves an imbalance of angiogenic factors, which leads to abnormal placentation, marked systemic inflammatory responses, and hypertension. One particularly relevant feature of this disease is the loss of the nocturnal decrease in arterial blood pressure. The authors assess the question of whether disruption of circadian function may represent an occupational risk factor for preeclampsia and make the case that while this condition should be considered a major public health concern, the evidence for shift work increasing the risk of preeclampsia is not yet persuasive enough to draw any solid conclusions. Using a DNA microarray approach, Tasaki et al. (2) analyzed gene expression in uterine endometrial stromal cells during the implantation stage in the rat. In particular, they focused on the evaluation of genes compromising the circadian clockwork as well as downstream clock-controlled genes, since mutations in the former are known to associate with implantation failure and other reproductive defects. Among their findings was evidence for the clock gene Bmall controlling inhibin- $\beta$, IGF-1, and other endometrial genes. They also developed the hypothesis that alterations in the circadian system during decidualization are important for regulating the expression of genes vital for the formation of the placenta. In his review, Sellix (3) highlights our current understanding of circadian clock functions in the female reproductive system as it relates to the timing of gonadotropin secretion at ovulation and the timing of parturition. He proposes that the neuroendocrine-reproductive axis should be considered a partnership of synchronized and cooperative oscillators whose disruption can help to explain some forms of fertility disorders. In their contribution, Gamble et al. (4) examine the importance of circadian clocks for successful pregnancy. It is well known that shift work increases the risk of miscarriages, preterm birth, low birth weights, etc. These authors identify key gaps in our knowledge of circadian clock function in reproductive physiology and offer useful strategies for better understanding how shift workinduced circadian misalignment leads to reproductive dysfunctions. The complex relationship between cholesterol metabolism, estrogens, and female reproductive cycles is reviewed by Urlep and Rozman (5). For example, various genes in the cholesterol and steroidogenic pathway are rhythmic and are regulated by clock genes. Conversely, the central circadian clock in the suprachiasmatic nuclei (SCN) expresses the estrogen receptor, ER $\beta$, which is also under clock gene control. Thus, perturbations in clock gene expression can have profound effects at multiple levels of the neuroendocrine-reproductive axis. Watanabe et al. (6) address the growing recognition that circadian rhythms during pregnancy have important consequences on fetal development. In their interesting clinical trials, they have determined that preterm babies (week 31 of pregnancy) already have pupillary reflexes and that exposure of these infants to a regular light/dark cycle (as opposed to the more common constant light environment of neonatal units) promotes more daytime activity and body growth. In other words, the direct use of natural circadian cycles of light and dark would appear to offer the preterm infant a surrogate rhythmic environment more conducive to optimal postnatal development, underscoring the importance of the circadian system during pregnancy. While it has long been recognized that female reproductive cycles are under the control of the central circadian clock in the SCN, only in the last few years have the specific details of this hypothalamic regulation been clarified. Miller and Takahashi (7) provide a lucid overview of the contributions of circadian vasopressin signals to the kisspeptin neurons that regulate hypothalamic GnRH secretion as well as the VIP regulation of pituitary prolactin rhythms. They also make a compelling case for the effects of clock gene mutations on pregnancy having important similarities to the impact of senescence on reproductive abnormalities.

As documented by this Research Topic, the study of cellular circadian clocks on reproductive systems is still fertile territory for endocrinologists, cell biologists, and clinicians alike. It has great potential for providing testable hypotheses and unified explanations for disparate processes, such as blood pressure and gene expression rhythms, effects of shift work and artificial lighting on fetal development, hormonal rhythms and fertility, etc. An exciting era awaits!

\section{REFERENCES}

1. Ditisheim AJ, Dibner C, Philippe J, Pechère-Bertschi A. Biological rhythms and preeclampsia. Front Endocrinol (2013) 4:47. doi:10.3389/fendo.2013.00047

2. Tasaki H, Zhao L, Isayama K, Chen H, Yamauchi N, Shigeyoshi Y, et al. Profiling of circadian genes expressed in the uterus endometrial stromal cells of pregnant rats 
as revealed by DNA microarray coupled with RNA interference. Front Endocrinol (2013) 4:82. doi:10.3389/fendo.2013.00082

3. Sellix MT. Clocks underneath: the role of peripheral clocks in the timing of female reproductive physiology. Front Endocrinol (2013) 4:91. doi:10.3389/fendo.2013. 00091

4. Gamble KL, Resuehr D, Johnson C. Shift work and circadian dysregulation of reproduction. Front Endocrinol (2013) 4:92. doi:10.3389/fendo.2013.00092

5. Urlep Z, Rozman D. The interplay between circadian system, cholesterol synthesis, and steroidogenesis affects various aspects of female reproduction. Front Endocrinol (2013) 4:111. doi:10.3389/fendo.2013.00111

6. Watanabe S, Akiyama S, Hanita T, Li H, Nakagawa M, Kaneshi Y, et al. Designing artificial environments for preterm infants based on circadian studies on pregnant uterus. Front Endocrinol (2013) 4:113. doi:10.3389/fendo.2013.00113

7. Miller BH, Takahashi JS. Central circadian control of female reproductive function. Front Endocrinol (2014) 4:195. doi:10.3389/fendo.2013.00195
Conflict of Interest Statement: The author declares that the research was conducted in the absence of any commercial or financial relationships that could be construed as a potential conflict of interest.

Received: 22 May 2014; accepted: 11 July 2014; published online: 24 July 2014.

Citation: Olcese J (2014) Circadian clocks and pregnancy. Front. Endocrinol. 5:123. doi: 10.3389/fendo.2014.00123

This article was submitted to Systems and Translational Endocrinology, a section of the journal Frontiers in Endocrinology.

Copyright $\odot 2014$ Olcese. This is an open-access article distributed under the terms of the Creative Commons Attribution License (CC BY). The use, distribution or reproduction in other forums is permitted, provided the original author(s) or licensor are credited and that the original publication in this journal is cited, in accordance with accepted academic practice. No use, distribution or reproduction is permitted which does not comply with these terms. 